FOLIA SCANDINAVICA VOL. 20 POZNAŃ 2016 DOI: $10.1515 / \mathrm{fsp}-2016-0042$

DE DE GRUYTER OPEN G

PRESSto.

\title{
ZUM TERMINOLOGISCHEN STATUS DER INKORPORATION IN DER DEUTSCHEN UND DER DÄNISCHEN WORTBILDUNGSBESCHREIBUNG
}

\author{
JANUSZ STOPYRA \\ Univeristy of Wroctaw
}

ABSTRACT. The article deals with the subject of incorporation shown as a special kind of composition and derivates formed from phrases (Zusammenbildungen). The author attempts to differentiate between these two types and also describes such phenomena as predicateargument-structure and the principle of the meaningful word. In his opinion it is worth using such terms to give the language description a better overview.

\section{DER TERMINUS INKORPORATION}

Den Terminus Inkorporation in dem im vorliegenden Aufsatz gebrauchten Sinne hat nach meinem Wissen als erster Eichinger (2000:156f) eingeführt. In der Tat handelt es sich um das bereits seit Jahrzehnten, besonders von Willmanns (1930) und Renicke (1965) behandelte terminologische Problem der Wortbildungsbeschreibung, das der letztgenannte Forscher gar als „das enfant terrible der deutschen Wortbildungslehre“ bezeichnet. Gemeint seien hier die sogenannten Zusammenbildungen und Rektionskomposita, die in die deutschsprachige Wortbildungsbeschreibung in Polen Rajnik (1974, 1983, 2004) eingeführt hat. Die Problematik wurde zusätzlich kontrastiv zur analogen sprachlichen Erscheinung des Dänischen besprochen. Die aus dem Wesen des Phänomens resultierenden terminologischen Schwierigkeiten sind dank seinen Werken, insbesondere nach ihrem Vergleich mit den Untersuchungen am dänischen Material und den dort dazu geäußerten Ansichten, klar geworden. Im Bereich der deutschsprachigen Wortbildungsbeschreibung in Deutschland hat das Phänomen ganzheitlich Leser (1990) zu behandeln versucht. 


\section{REKTIONSKOMPOSITA UND ZUSAMMENBILDUNGEN}

Es handelt sich dabei um die Wortbildungskonstruktionen vom Typ $\mathrm{M} 1+\mathrm{M} 2+\mathrm{M} 3$, die jeweils drei Morpheme enthalten $(\mathrm{M}=$ Morphem), von denen zwei erstere Grundmorpheme und das dritte (M3) zumeist ein Wortbildungsmorphem darstellen kann. Es gibt zwei Hauptmuster der Inkorporationen, und zwar die vom Typ Adjektiv + Substantiv + M3 oder Substantiv + Verb + M3. Wenn die Morphemkombination M2+M3 ein frei vorkommendes Wort darstellt, haben wir es mit Rektionskomposita, sonst mit Zusammenbildungen zu tun.

Die ganzheitliche Behandlung der beiden Phänomene in der Wortbildungsbeschreibung resultiert daraus, dass sich derartige Bildungen häufig als polysem und damit zweideutig, d. h. entweder als Zusammenbildungen oder als Rektionskomposita, bezeichnen lassen. Die meisten der terminologischen Probleme bereiten dabei die Zusammenbildungen, mit denen wir es also in dem Falle zu tun haben, wenn die o g. Bestandmorpheme M2+M3 nicht frei als ein ganzes Wort auftreten.

Dank Rajnik (1974, 1983, 2004) hat die deutsche und dänische Wortbildungslehre nicht nur eine genaue Beschreibung dieses Phänomens, sondern auch seine ganzheitliche Erklärung gewonnen. Der Forscher teilt die Zusammenbildungen in feste und unfeste, wobei die ersteren v. a. präfigierten Verben (vom Typ begünstigen) gleichzusetzen sind. Sein Hauptinteresse und damit auch den Kern des Problems stellen aber die unfesten Zusammenbildungen dar, die anschließend eingehend beschrieben und erklärt worden sind. Dabei gebraucht der Autor eine präzise Metasprache, die das Vermächtnis seiner Studien der generativen Transformationsgrammatik darstellt. Wenn man also den in der Wortbildungsbeschreibung bekannten Wettbewerb zwischen ihrem lexikalistischen und syntaktischen Ansatz verfolgt, so ist das Schaffen von Rajnik (1974, 1983, 2004) dem syntaktischen Paradigma zuzuordnen.

Besonders interessant sind diejenigen Erkenntnisse des Autors, die aus dem Vergleich der deutschen und der dänischen Wortbildungsbeschreibung aus dem Bereich der Inkorporation resultieren. In der letzteren finden wir häufig auch alternative Sehweisen vor, die in der deutschen Wortbildungsbeschreibung nicht anzutreffen sind. Nach einer von solchen Formulierungen werden die dänischen Possessivkomposita als solche angesehen, die Zusammenbildungen ohne Suffix (dänisch: samdannelser uden suffiks) darstellen. Das Interesse, das diesem analog im Deutschen und im Dänischen präsenten Phänomen gilt, kann seitens der dänischen Wortbildungsbeschreibung als häufiger als seitens der deutschen bezeichnet werden.

Das Phänomen der Inkorporation wird von den Forschern in der Regel bemerkt, es herrscht unter ihnen jedoch keine Einstimmigkeit darin, dass es als mit einem gesonderten Termin 'Zusammenbildung' erfasst zu werden verdient 
(während das Vorhandensein von Rektionskomposita in der Regel nicht negiert wird). Kein Problem besteht im Falle, wenn die o. g. Morpheme M2+M3 als ein frei vorkommendes Wort üblich sind. In dem Falle haben wir es mit Rektionskomposita zu tun, vgl. Taxifahrer, Gewichtheber (s. u.). Die terminologischen Schwierigkeiten beginnen aber dort, wo sich bei den betrachteten Bildungen keine frei vorkommende autosemantische Zweitkonstituente, die aus den Elementen M2 + M3 aufgebaut wäre, heraussondern lässt. Derartige Wortbildungen werden in der Regel als Derivate von phrasalen Basen aufgefasst und als Zusammenbildungen (dänisch: samdannelser) bezeichnet. Belege wie z. B. Gesetzgebung - Gesetz geb(en) + -ung, Grundsteinlegung Grundstein leg(en) + -ung (dänisch taksigelse - (at) sige tak + -else, lovgivning - (at) give lov + -else), wo zu einer gegebenen Wortbildung eine jeweils gebräuchliche Phrase existiert, erlauben es, sie tatsächlich auf solche Weise, d. h. als Ableitungen mit einer Phrase als Basis, zu klassifizieren (so z. B. Fleischer/Barz (1995:47), die die Notwendigkeit der Einführung der ,Zusammenbildung' als gesonderten Termins zurückweisen). Die terminologischen Entscheidungen verkomplizieren aber Bildungen wie z. B. Bittsteller, Bewegungsmelder, zu welchen sich nicht allzu strickt eine Paraphrase bilden lässt, die genau in das terminologische Raster der Ableitungsbasis für diese Bildungen passen würde (*Bitte stellen, *Bewegung melden).

Die mit Abstand zahlreichste Gruppe von Zusammenbildungen stellen die Nomina agentis auf -er, die sich zugleich auch als Nomina instrumenti interpretieren lassen, z. B. Hosen-, Gepäckträger, Filmemacher, Taxi-, Milchfahrer, Machthaber usw. Häufig können sie, wie bereits anfangs angekündigt, auch als polyseme Rektionskomposita eingestuft werden. Wenn man aber davon ausgeht, dass Wörter wie *Träger, *Macher im Gegenwartsdeutschen ungebräuchlich sind, muss man die entsprechenden sie enthaltenden Wortbildungen als Zusammenbildungen einstufen.

Äußerst interessant sind die dabei vorkommenden Unterschiede zwischen dem Deutschen und dem Dänischen, die die Frage nach dem Ausmaß und den Grenzen für die deutsch-dänische Wortbildungsparallelität teilweise beantworten lassen. Nach $D D O$ werden manche derartige Bildungen des Dänischen, die gegenüber den deutschen als semantisch und formal äquivalent zu bezeichnen sind, als solche dargestellt, wo sich, im Gegensatz zum deutschen Äquivalent, jeweils eine entsprechend aus den Morphemen M2 und M3 aufgebaute, gebräuchliche Zweitkonstituente heraussondern lässt, z. B. jagger in ordjager (zu deutsch Wortjäger). Nach $D D O$ werden derartige Zweitkonstituenten im Dänischen häufiger als gebräuchlich zugelassen als nach entsprechenden Wörterbüchern des Deutschen.

Die Überhand in der deutschen und dänischen Wortbildungsbeschreibung gewinnen Meinungen, nach welchen man die betrachtete Menge von Bildungen mit einem gesonderten Termin versehen sollte. Es fehlt jedoch auch 
nicht an Meinungen, wonach sie sich entweder als Zusammensetzungen oder Derivate terminologisch interpretieren ließen. Nach meiner Meinung hängt alles davon ab, von welcher Definition der Zusammensetzung man bei dieser Entscheidung ausgeht. Nach Morciniec (1964) stellen die Zusammensetzungen Gefüge von selbstständigen, unmittelbar aufeinander folgenden Konstituenten dar, deren Entfernung voneinander nicht variieren kann und deren Reihenfolge bedeutungsrelevant ist. Unsere Hauptfrage sollte demnach nach dem Terminus 'selbstständig' (von der o. g. Definition) gestellt werden. Nach Morciniec (1964) heißt 'selbstständig' zugleich austauschbar, was man nach meiner Meinung als 'mit der Möglichkeit, frei im Satz vorzukommen' bezeichnen könnte. Nach Annahme dieser Definition ist somit von Zusammensetzungen als solchen Wortbildungskonstruktionen auszugehen, deren unmittelbare Konstituenten frei im Satz vorkommen können. Dies lässt uns also an der Diskussion um die Zusammenbildungen beteiligen. Die Tatsache, dass Bildungen wie *Seher, *Hörer nicht als freie Zweitkonstituenten vorkommen, d. h. nicht sprachüblich sind, erlaubt uns nicht, das betrachtete Phänomen als Zusammensetzungen zu bezeichnen und lässt uns auf die Suche nach anderen terminologischen Lösungen begeben.

Nach der Popularisierung des Problems von Rajnik (1974, 1983, 2004) haben auch weitere Forscher begonnen, sich mit dieser Thematik zu interessieren. Ein Teil der Bearbeitungen wurde in Stopyra (2008:99ff.) dargestellt, die vorliegende Studie nimmt die Beschreibung weiterer Werke vor. Generell kann von Rektionskomposita dann die Rede sein, wenn als eines ihrer Bestandmorpheme ein transitives Verbstamm vorkommt. Als mögliche Suffixe kommen im Deutschen am häufigsten -er, -ung, -ig und $-e$, im Dänischen -ing, -else, -er, -ig und - et vor. Die Autoren führen für die Inkorporationen verschiedene Belege an: Frauenkenner, Romanleser, Konfliktbewältigung, Wetterbeobachtung als Rektionskomposita und Dickhäuter, Appetithemmer, Schwarzseher, Langschläfer, Frühlingsblüher, Grablegung, Vogelscheuche, blauäugig, viertürig als Zusammenbildungen bei Donalies (2007:45ff.); die Autorin folgt jedoch der Theorie von Leser (1990), indem sie von der Nennung der Letzteren als Zusammenbildungen zugunsten von ,Kombinationen aus frei vorkommenden Wörtern, deren freies Vorkommen argumenterblich beschränkt wird“ (Belege Vogelscheuche, Frühlingsblüher und Appetithemmer) oder zugunsten von ,Kombinationen aus Phrasen“ (die Belege mit Adjektiv als M1) absieht; weiterhin Schuhmacher und Kampfführung als Zusammenbildungen und friedensbereit, lawinensicher, gegenwartsfern, heroinsüchtig, berufstauglich, frauenfeindlich als Rektionskomposita bei Lohde (2006:80, 159); Bergsteiger, Staubsauger, Teilhaber, Liebhaber, Machthaber, Kriegstreiber, Ski-, Amokläufer, Bahnbrecher, Ehebrecher, Eckensteher, Zechpreller, Schönfärber, Schnellläufer, Schallgeber, Arbeitnehmer, Reifenheber, Mähbinder, dreieckig, friedliebend, breitschultrig, ebenerdig, erfolgs-, 
vorwärts-, zielstrebig, grob-, sanft-, feinfühlig, locker-, leichtlebig, vier-, ganztägig, zehnjährig, beidäugig, -armig, -ohrig, -füßig, -fäustig, spitzmundig, linksarmig, eigenhändig, als Zusammenbildungen und Kolbenschieber, Flachschieber, Uhrzeiger als Rektionskomposita bei Motsch (2004:9 et passim); Schnelldurchblicker, Frauenversteher, Rückwärtseinparker, Wunderwirker, Trübsinnhabachter, Farbgebung, Inbetriebsetzung, Rechtshänder, Stellungnahme, Liebhaber, Ichübergreifung, Sozialverekler als Zusammenbildungen und Busfahrer, Erkundlehrer, Hausbewohner, Kinobesucher, Nussknacker, Wasserträger, Weintrinker, Buchbinder als Rektionskomposita bei Elsen (2011:43 et passim), vgl. dänisch ihoendehaver, arvtager, modstander, lovgivning, madlavning, -dyrkning, tilkendegivelse, tykmavet, bredskuldret als Zusammenbildungen und natvagter, gavegiver, rådgiver, arbejdsgiver, soldyrker, ordforer, kraftbekampelse, lodtrakning, todeling, als Rektionskomposita usw. Diese sämtlichen Belege, insbesondere die von Elsen (2011) sind häufig im täglichen Gebrauch. Das gesamte Phänomen wird am treffendsten aber bei Simmler (1998) zusammengefasst.

Der von Simmler (1998:410) zitierte Beitrag von Renicke (1965:45) betrachtet Belege wie Nussknacker und Wasserträger nicht als Determinativkomposita, weil hier nicht Knacker und Träger determiniert werden, sondern das ,,vorkompositionelle Rektionsverhältnis (Aussagegröße - Beziehungsgröße) verdichtet in die Zusammenfügung eingegangen“ ist. Die Zusammenbildungen wie Wichtigtuer lassen uns danach als die zwei ersteren Morpheme (M1+M2) eine Morphemkonstruktion bemerken, die nicht als eine vollständige gängige Phrase (bei Simmler: Wortgruppe) - wie sie z. B. bei den Zusammenrückungen erkennbar ist - funktioniert. Sie ergibt sich erst aus einer zugrunde liegenden Paraphrase. Nach dem von Simmler zitierten Beitrag von Höhle (1982) stellen die Zusammenbildungen Komposita dar, die ,nicht wie gewöhnlich frei als Wort vorkommen, sondern nur als Bestandteil eines anderen Wortes“ (nach Simmler, 1998:410). Letzten Endes spricht sich Simmler (1998:410) für die Beibehaltung im Falle von Belegen wie Liebhaber des Termins Zusammenbildungen, weil derartige Bildungen weder als Komposita noch als Derivate erfasst werden können. Nach Simmler kann man angesichts der Anwesenheit dieses spezifischen sprachlichen Phänomens nicht davor terminologisch zurückweichen, sondern es mit einem gesonderten Termin sanktionieren. Simmler definiert die Zusammenbildung somit als „das Ergebnis eines Wortbildungsprozesses, bei dem auf der Grundlage einer Wortgruppe Prinzipien der Komposition und Derivation gleichzeitig zusammenwirken, um ein neues Wort zu konstituieren, dessen ausdrucksseitige interne morphologische Strukturen und inhaltsseitige Beziehungsgefüge den Bildungsprinzipien von Determinativkomposita mit einem mit demselben Formationsmorphem [d. h. Wortbildungsmorphem] gebildeten Zweitglied entsprechen, wobei ein klares zeitlichen Nacheinander von Wortgruppe und 
einer Worteinheit existiert.“ Als einziger spricht Simmler (1998) also von einem zeitlichen Nacheinander der Phrase und der auf ihrer Grundlage geprägten Zusammenbildung.

\section{ZUR ARGUMENTVERERBUNG}

$\mathrm{Zu}$ äußerst interessanten Phänomenen, die bei den Zusammenbildungen / Rektionskomposita vorkommen, gehören die Argumentvererbung und das Prinzip des sinnvollen Wortes. Die Argumente des Verbs als M2 perkolieren demnach auf das ihm angehängte Suffix, und infolgedessen werden sie zu Argumenten des Derivats M2+M3 (das gegebenenfalls nicht sprachüblich zu sein braucht), jedoch häufig mit verändertem Status (d. h. z. B. als fakultativ an Stelle von obligatorisch), wonach sie links daran inkorporiert werden, vgl. 'jmd. fährt Milch' > ,Fahrer von Milch' > Milchfahrer, , at bære bagage' > ,bærer af bagage' > bagagebcerer (vgl. Stopyra, 2008:100ff). Die Argumentvererbung tritt gleichermaßen im Falle sowohl von Rektionskomposita als auch von Zusammenbildungen auf, nur ist der Status des Derivats (M2+M3) als „Zwischenstufe“ bei den letzteren als nicht sprachüblich zu bezeichnen.

\section{ZUM PRINZIP DES SINNVOLLEN WORTES}

Das Prinzip des sinnvollen Wortes wurde als solches von Motsch (2004:26) ausformuliert. Danach sind Derivate wie *beinig, *äugig, *händig usw., im Gegensatz zu bucklig, bärtig, kommunikativ sinnlos, weil sie „kommunikative Gemeinplätze“ bezeichnen (weil alle Hände, Augen, Beine usw. haben). Sie können jedoch ,verfügbar gemacht werden“, indem sie, dank der Argumentvererbung, ein entsprechendes Argument des Verbs (Hier: haben) links versetzt bekommen, d. h. einbeinig, einäugig, einhändig usw., wobei Bildungen wie z. B. *zweiäugig aus sprachpragmatischen Gründen wiederum unüblich sind (weil alle zwei Hände, Beine Augen usw. haben). Hier tritt aber als nächste die Möglichkeit auf, allgemein als untypisch geltende menschliche Eigenschaften als sprachübliche Wörter nach dem o. g. Prinzip zu konstruieren, d. h. z. B. linkshändig (im Gegensatz zu *rechtshändig); vgl. dänisch enøjet, enbenet, kejthåndet usw.

\section{ZUSAMMENFASSUNG}

Die Phänomene wie Rektionskomposita und Zusammenbildungen, bei denen die strukturell-funktionale Ambivalenz von Komposition und Derivation eine sie konstituierende Eigenschaft darstellt, sind zumeist interpretativ eindeutig: auch ohne Kontext stellen sie, auch als neu geprägte Augenblicks- 
bildungen, in der Regel semantisch eindeutige Wortbildungen dar (außer Ausnahmen wie Alkoholfahrer - 'Fahrer von Alkohol' oder 'Fahrer unter dem Einfluss von Alkohol' - sowie außer scherzhaft gemeinten Bildungen wie Kaiserjäger - ,Jäger des Kaisers, d. h. der dem Kaiser untertan ist' oder 'einer, der den Kaiser jagt' bei Eichinger, 2000). So stehen sie unter diesem Gesichtspunkt in Opposition zu den Komposita, die, abhängig vom Kontext und der Sprechsituation, eine theoretisch beinahe unbegrenzte Zahl von möglichen Deutungen haben können (vgl. das Beispiel Fischfrau bei Heringer, 1984a:1ff).

Was von vielen Forschern unerwähnt bleibt, ist die Möglichkeit, die Morphemverbindung (M1+M2+M3), außer ihrer Deutung als Rektionskomposita und Zusammenbildungen, auch als Ableitungen von Zusammensetzungen $\mathrm{zu}$ interpretieren, z. B. viereckig (zu Viereck), reiselustig (zu Reiselust), Handwerker (zu Handwerk), dänisch firkantet (zu firkant), rejselysten (zu rejselyst), håndvarker (zu håndvark).

Wiewohl der Terminus 'Zusammenbildung / samdannelse' von manchen Forschern abgelehnt wird, trägt er einerseits dem Vorhandensein eines bestimmten sprachlichen Phänomens Rechnung, andererseits verschafft er dank seiner Prägnanz eine größere Übersichtlichkeit in Wortbildungssystemen der untersuchten Sprachen (im Vergleich zu etwa ,Kombinationen aus Phrasen“ von Donalies, 2007:45ff). Im Großen und Ganzen lässt sich die anfangs von Rajnik (1974, 1983, 2004) angesprochene Problematik jetzt als ein häufiger Diskussionsgegenstand bezeichnen, der, angesichts der großen Popularität des besprochenen Wortbildungsmusters, gar als der Existenz einer sprachlichen Mode Rechnung tragend bezeichnet werden kann. Damit kann zugleich auch die Meinung widerlegt werden, wonach das wissenschaftliche Paradigma der Wortbildung bereits erschöpft sei ${ }^{1}$.

\section{LITERATUR}

Diderichsen, P: (1946). Elementer dansk Grammatik. København Gyldendalske Boghandel, Nordisk Forlag A/S.

Donalies E. (2007): Basiswissen Deutsche Wortbildung. Tübingen/Basel: A. Francke Verlag. $D D O=$ Den Danske Ordbog. I-VI. København: Det Danske Sprog og Litteraturselskab, Gyldendal.

Eichinger, L. M. (2000). Deutsche Wortbildung. Eine Einführung (Narr Studienbücher). Tübingen: Narr Verlag.

Elsen, H. (2011). Grundzüge der Morphologie des Deutschen. Berlin/Boston: Walter de Gruyter. Fleischer, W., Barz, I. (1995). Wortbildung der deutschen Gegenwartssprache. Tübingen: De Gruyter. Hansen, Aa. (1967). Moderne Dansk I-II. København:Grafisk Forlag.

Hansen, E., Heltoft, L. (2011): Grammatik over det Danske Sprog. København: Det Danske Sprog og Litteraturselskab.

Heringer, H. J. (1984a). Wortbildung: Sinn aus dem Chaos. In: Deutsche Sprache 1, S. 1-13.

Heringer, H. J. (1984b): Gebt endlich die Wortbildung frei! In: Sprache und Literatur in Wissenschaft und Unterricht 15, S. 43-53.

\footnotetext{
${ }^{1}$ Hörbeleg, vernommen an der Universität Wrocław, 2012.
} 
Höhle T. N. (1982). Über Komposition und Derivation: zur Konstituentenstruktur von Wortbildungsprodukten im Deutschen. Zeitschrift für Sprachwissenschaft 1, S. 76-112.

Leser, M. (1990). Das Problem der 'Zusammenbildungen'. Eine lexikalistische Studie (=FOKUS. Linguistisch-Philologische Studien, Band 3). Trier: Wissenschaftlicher Verlag.

Lohde, M. (2006). Wortbildung des modernen Deustchen. Ein Lehr- und Übungsbuch. Tübingen: Narr Studienbücher.

Morciniec, N. (1964). Die nominalen Wortzusammensetzungen in den westgermanischen Sprachen (= Prace Wrocławskiego Towarzystwa Naukowego, Serie A, Nr. 99). Wrocław: Zakład Narodowy im. Ossolińskich.

Motsch, W. (2004). Deutsche Wortbildung in Grundzügen (=Schriften des Instituts für Deutsche Sprache 8). Berlin, New York: De Gruyter.

Rajnik, E. (1974). Zum Bestand und zur Struktur der Zusammenbildungen im Deutschen. Studia Germanica Posnaniensia III, S. 87-104.

Rajnik, E. (1983). Unfeste substantivische Zusammenbildungen im Deutschen und Dänischen. In: Zeszyty Naukowe Wydziału Humanistycznego Uniwersytetu Gdańskiego. Studia Scandinavica 6, S. 71-105.

Rajnik, E. (2004). Adjektiviske samdannelser i moderne dansk. In: H. Jørgensen, P. S. Jørgensen (red.). PÅ GODT DANSK. Festskrift til Henrik Galberg Jacobsen i anledning af hans 60 års fødselsdag 4. februar 2004 (S. 223-230). Aarhus: Wessel og Huitfeldt.

Rajnik, E. (2011). Wortbildung des Substantivs im Dänischen - explizite und implizite Derivation. Poznań: Wydawnistwo Naukowe UAM.

Renicke, H. (1965). Fragen der Kompositionslehre. In: Zeitschrift für deutsche Philologie 84, S. 409-419.

Simmler, F. (1998). Morphologie des Deutschen. Flexions- und Wortbildungsmorphologie (=Germanistische Lehrbuchsammlung 4). Berlin: Weidler Buchverlag, 355-640.

Stopyra, J. (2008). Nominale Derivation im Deutschen und im Dänischen. Wrocław: Wydawnictwo Uniwersytetu Wrocławskiego.

Stopyra, J. (2012). Inkorporation in der nominalen Wortbildung (am Beispiel des Deutschen und Dänischen). In: P. Chruszczewski, S. Prędota (Hrsg.). Prace Komisji Nauk Filologicznych Oddziału Polskiej Akademii Nauk we Wroctawiu II. Polska Akademia Nauk, Oddział we Wrocławiu, Wrocław 2010, S. 147-157.

Stopyra, J. (2013). Überschaubarkeit in der Wortbildung: Uneigentliche Komposita und Rektionskomposita. In: I. Bartoszewicz et al. (Hrsg.), Im Anfang war das Wort II. Linguistische Treffen in Wrocław 9 (S. 265-270). Wrocław/Dresden: ATUT-Neisse Verlag.

Szubert, A. (2012). Zur internen Semantik der substantivischen Komposita im Dänischen. Poznań: Wydawnictwo Naukowe UAM.

Willmanns, W. (1930). Deutsche Grammatik. Gotisch, Alt-, Mittel- und Neuhochdeutsch. Zweite Abteilung: Wortbildung. Berlin-Leipzig: Walter de Gruyter.

\section{INTERNETQUELLEN}

www.ids-mannheim.de [29.09.2016]

www.dsl.dk [29.09.2016]

\section{Janusz Stopyra}

Uniwersytet Wrocławski

Instytut Filologii Germanńskiej

pl. Nankiera $15 \mathrm{~b}$

50-140 Wrocław

Poland

janusz.stopyra@uwr.edu.pl 